\title{
GIOVANNI PERES
}

Osservatorio Astronomico di Palermo, Piazza del Parlamento 1, I-90134 Palermo, Italy

\begin{abstract}
This paper discusses the hydrodynamic modeling of flaring plasma confined in magnetic loops and its objectives within the broader scope of flare physics. In particular, the Palermo-Harvard model is discussed along with its applications to the detailed fitting of X-ray light curves of solar flares and to the simulation of high-resolution CaXIX spectra in the impulsive phase. These two approaches provide complementary constraints on the relevant features of solar flares. The extension to the stellar case, with the fitting of the light curve of an X-ray flare which occurred on Proxima Centauri, demonstrates the feasibility of using this kind of model for stars too. Although the stellar observations do not provide the wealth of details available for the Sun, and, therefore, constrain the model more loosely, there are strong motivations to pursue this line of research: the wider range of physical parameters in stellar flares and the possibility of studying further the solar-stellar connection.
\end{abstract}

\section{Introduction}

Hydrodynamic loop models of flares aim at calculating the evolution of confined plasma during the thermal phase of flares, under simplifying hypotheses. They achieve a reasonable level of detail and of realism by describing the simultaneous influence of the effects dominating the thermal phase of flares (e.g., thermal conduction, compressible hydrodynamics, radiative losses), in the fully non-linear regime and in the loop-like geometry shown by observations.

Their scope covers only some aspects of flares but, on the other hand, a complete theory of flares, describing all the phenomena and their intricate interaction, has never been developed. Most of the phenomena consider to be the primary cause of the flare, like magnetic field dissipation, MHD instabilities, electron acceleration etc, have been the object of a considerable amount of theoretical work but there are too few direct data on them. Most of the secondary effects, like plasma motion, hard X-rays, soft X-ray light curves and line profiles, are just a consequence of the primary effects; yet they have been observed with a much higher level of detail.

Hydrodynamic models of flares can provide a link between the well-observed thermal phase, characterized by the secondary effects, and the primary effects. After (and if) the model has provided a satisfactory reproduction of the observations, one can impose constraints on the primary mechanisms using the parameters of the model yielding the best agreement. For instance the localization and extension of the heating function used in the model, as well as the timing required to fit a flare, might give information on the primary energy release mechanism. Analogously the computed maximum plasma pressure provides a lower limit on the magnetic field needed to confine the plasma. Of course some diagnostic power lies also in the possibility of proving the ineffectiveness of some specific mechanism. This approach evidences the diagnostic possibilities of hydrodynamic models, an interesting expansion of their use as theoretical tools. 
Several groups have developed models of this kind and used them to tackle several of the problems existing in solar physics (see references in Peres and Serio, 1984; in Kopp et al., 1986; and in Peres et al., 1987).

Although each of the different models has unique features, either in the treatment of the physical effects or in the numerical technique used, they share many similarities. In order to illustrate their possibilities in more detail, I discuss below the features and some results on solar and stellar flares, of the Palermo-Harvard model, the one developed by my group.

\section{The Palermo-Harvard Model}

Space observations have shown the magnetic structuring of the whole solar atmosphere (for a review, see Vaiana and Rosner, 1978) and, more recently, the evidence of magnetic confinement of coronae in late-type stars (for a review, see Rosner, Golub, and Vaiana, 1985). Therefore, the thermal conduction and any fluid motion of the atmospheric plasma is channelled along the field lines and any closed magnetic loop can be modelled as a structure dynamically and thermodynamically independent from the neighbouring structures. Except for high-speed electrons in the transition region (see Section 5), the particle mean free path is shorter than the loop dimensions by orders of magnitudes and this permits a fluid treatment. Thermal flares have been shown to occur in closed loops (Pallavicini et al., 1975; Pallavicini, Serio, and Vaiana, 1977) whose morphology, for the case of compact flares, does not change significantly. Two-ribbon flares, instead, show evident changes in the loop morphology and, therefore, have a qualitatively different evolution.

Our model describes directly only the thermal phase of compact loop flares. It is based on the single fluid differential equations of mass, momentum and energy conservation, taking into account the effect of ionization, gravity, viscosity, heating, optically thin plasma radiative losses and thermal conduction along with the equation of state of the gas (Peres et al., 1982). The loop is assumed to be semi-circular, of constant cross section, to be infinitely rigid and anchored to the photosphere. The magnetic field plays no explicit role other than confining the plasma inside the coronal loop. The model is symmetric with respect to the loop apex and the initial atmosphere is static.

The heating function consists of two components: a steady heating, needed to maintain the atmosphere in static conditions by balancing the energy losses, and an impulsive heating which drives the flare by overheating the confined plasma. We have chosen two formulations of the impulsive heating. The first is a separable function of time and field-line coordinate:

$$
Q(s, t)=E_{H} \times f(t) \times g(s),
$$

where $E_{H}$ is the maximum intensity of the heating, $g(s)$ is a Gaussian whose center and spread can be chosen at will, and $f(t)$ yields the chosen evolution of the heating. The alternative formulation describes the effect of electron beams, precipitating from the loop apex to the loop footpoints, with a power-law energy distribution, of index $\delta$, 
abruptly truncated at the low-energy cutoff $E_{c}$, and of a given intensity and temporal evolution. In this approach we have followed the treatment of Nagai and Emslie (1984).

The equations are nonlinear and too complex to be solved analytically and, therefore, have to be solved numerically, by translating the differential equations to difference equations which in turn are solved by computer.

\section{Applications to the Solar Flares}

The fitting of the observations of the X-Ray Polychromator (XRP) on board the Solar Maximum Mission (SMM) satellite first showed the diagnostic possibilities of the model. This instrument measures the flux of seven X-ray lines (O vIII at $18.97 \AA$, Ne IX at $13.45 \AA, \mathrm{Mg}$ XI at $9.17 \AA$, SiXIII at $6.65 \AA, \mathrm{S}$ XV at $5.04 \AA$, Ca XIX at $3.17 \AA, \mathrm{FeXXV}$ at $1.85 \AA$ ) whose temperatures of maximum emissivity cover the range of temperature of the coronal flaring plasma (Acton et al., 1980).

Our approach was to synthesize the plasma emission from the evolution of density, temperature and velocity computed with the model, and then to compare it with the observations. We checked the general agreement of the model with the observations (Pallavicini et al., 1983), and found a few general results, such as the importance of the Fe Xxv line, among the observed ones, to trace the timing of the heating. We then turned to the detailed fitting of a specific flare observed in high level of detail by SMM and by Earth-based instruments (Peres et al., 1987a). We made the calculations using two models of local thermal heating (either localized at the loop apex, or at the base of the corona) and two models of heating by electron beams (either with $E_{c}=10 \mathrm{keV}$ or with $E_{c}=25 \mathrm{keV}$, and both with $\delta=8$ ). We performed several simulations for all heating models.

As an example of the results obtained, Figure 1 reports the best fitting cases for the heating with electron beams for $E_{c}=10 \mathrm{keV}$ and Figure 2 for $E_{c}=25 \mathrm{keV}$. The fitting is satisfactory for the first case but much less for the second. The implications are that in this flare, although high-energy electrons are present, as shown by the hard X-ray observations (MacNeice et al., 1985), they do not seem to drive the soft X-ray flare.

A rather dynamic evolution, with impulsive heating of the plasma, rise of plasma temperature, and chromospheric evaporation, characterizes the rise phase of the thermal flares. Diagnostics of this phase can be obtained with the high-resolution spectra of $\mathrm{X}$-ray lines such as that of Ca XIX around 3.17 $\AA$, taken by the Bent Crystal Spectrometer (BCS) on board SMM. Antonucci et al. (1982) proved that, during the early phase of flares, the main component of this line typically broadens and a blue-shifted component, taken as evidence of plasma moving toward the observer at hundreds of kilometers per seconds, becomes prominent. The analysis of spectra at different times provides the evolution of plasma velocity and temperature (Antonucci et al., 1982).

We are synthesizing CaXIX spectra from loop flare simulations under a wide range of hypotheses for the impulsive heating and for the pre-flare atmosphere, to understand their effect on the spectral features of typical flares observed with the BCS. So far we have completed the analysis of cases of thermal heating. Figure 3 shows two spectra, 

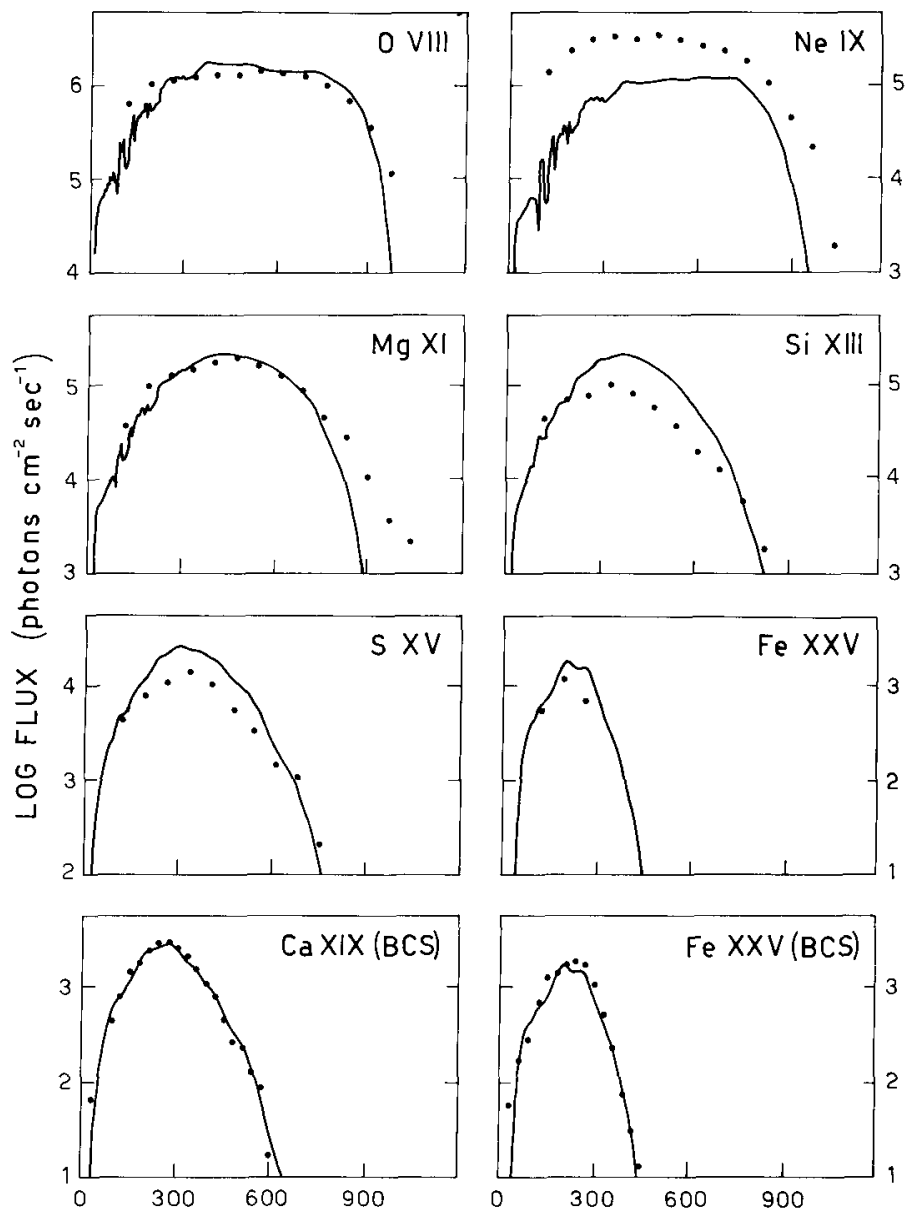

$T / M E($ sec)

Fig. 1. Modelling of the solar flare of November 12, 1980 at 17:00 UT: observed (dots) and computed (solid lines) light curves in the seven XRP lines. The flux is in photon $\mathrm{s}^{-1} \mathrm{~cm}^{-2}$ at Earth. In the simulation we assume heating by beams of non-thermal electrons with $E_{c}=10 \mathrm{keV}$ and $\delta=8$. (From Peres et al., 1988).

from Antonucci et al. (1987), computed with the Palermo-Harvard code for local thermal heating at the loop apex (Figure 3(a)), and for heating at the loop base (Figure 3(b)) in otherwise identical model loops. The case of heating at the footpoints reproduces features, like blue shifts, observed in at least $40 \%$ of the cases and, therefore, we can infer that impulsive heating in these flares most likely occurs near the loop footpoints rather than at the loop apex. On the other hand signatures for heating at the loop apex typically evolve rapidly and occur mostly at the beginning of the flare, when the emission is still rather weak. Since, at the beginning of the flare, present day instrumentation has to integrate over an extended time, any possible signature of heating at the loop apex is cancelled. 

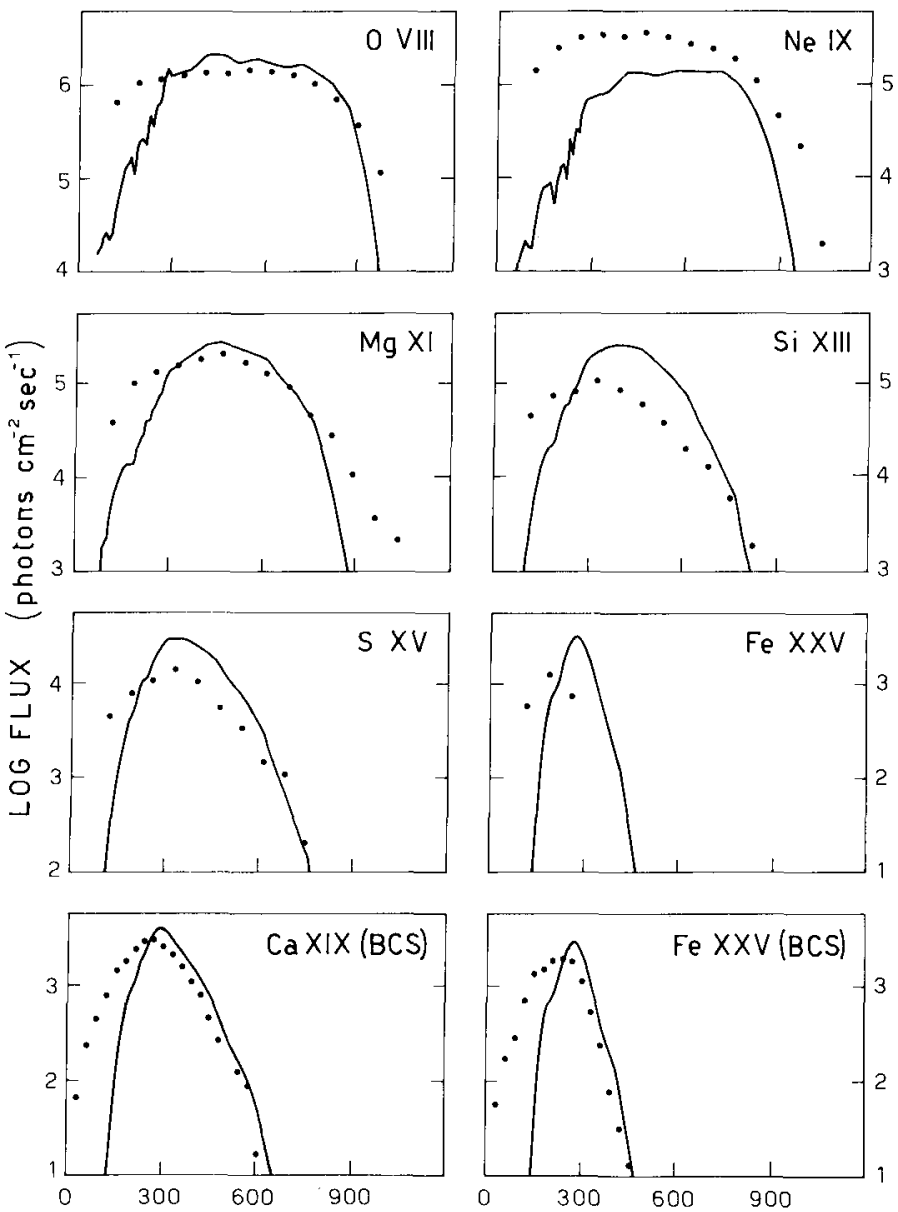

$$
\text { TIME (sec) }
$$

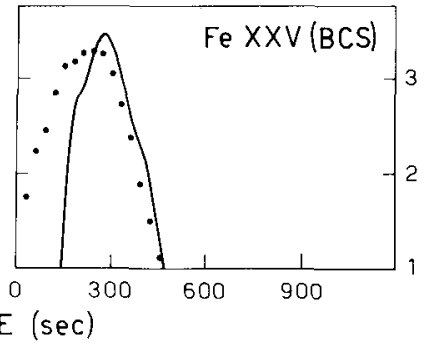

Fig. 2. As in Figure 1, but for $E_{c}=25 \mathrm{keV}$. (From Peres et al., 1987.)

\section{The Extension to Stellar Flares}

The satisfactory results obtained for the Sun have encouraged us to extend this technique to the stellar case to show similarities or differences between solar and stellar flares. Further interest lies in the wider range of physical parameters of the stellar environment but, on the other hand, the observation of events on other stars necessarily lack the fine details available for the Sun. Therefore, this novel approach aims also at constraining the dimensions of the flaring structure, and possibly to explore the dependence of magnetic field, of heating and of effective temperature on stellar characteristics.

Reale et al. (1988) have tackled the simulation of a flare which occurred on Proxima Centauri and was observed by the Einstein telescope (Giacconi et al., 1979) on August 20, 1980 (Haisch et al., 1983). The detector in the focal plane of the telescope was the Imaging Proportional Counter. Figure 4(a) reports the light curve as seen by the Imaging 


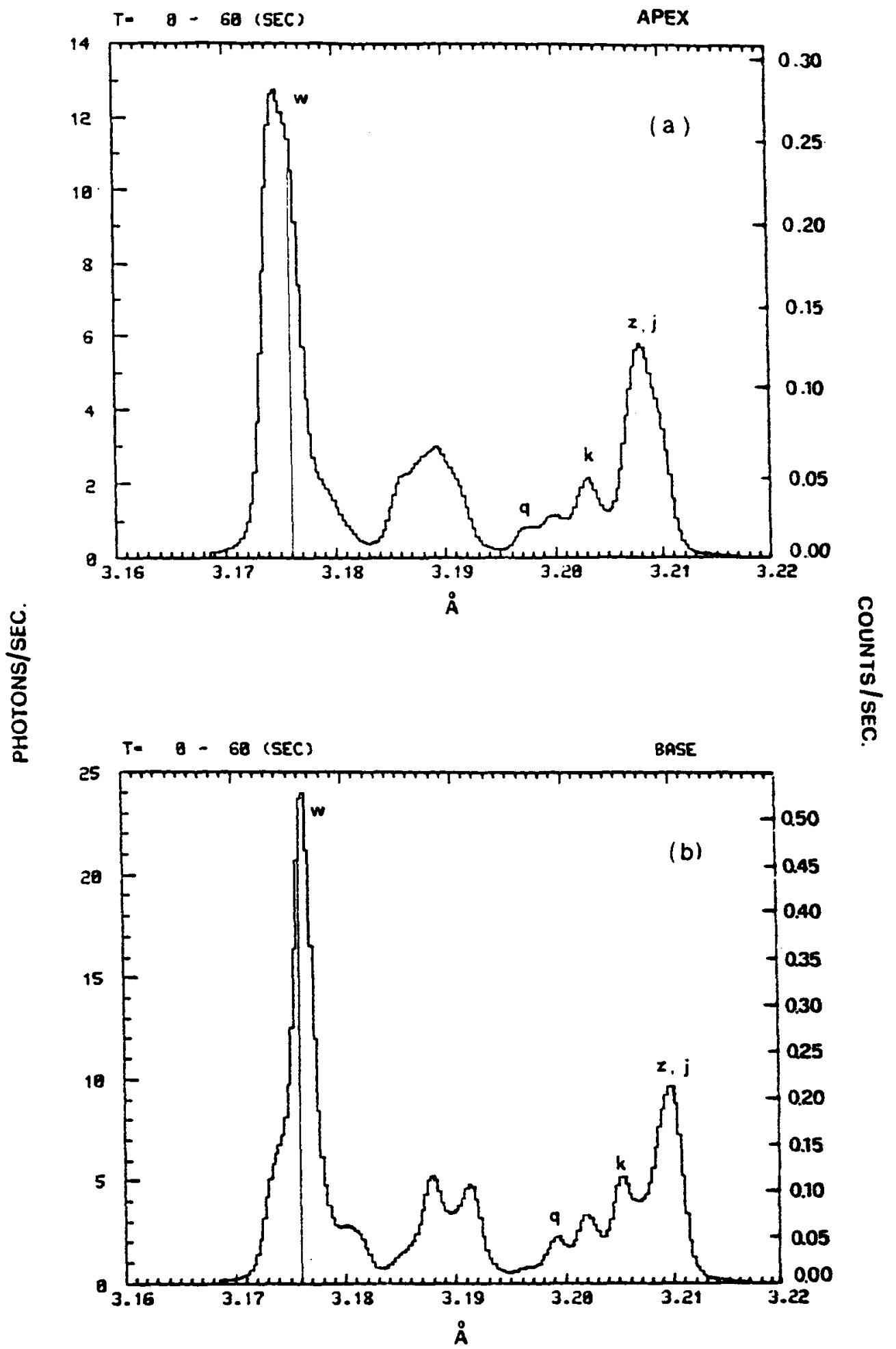

$\frac{j}{\frac{1}{0}}$

Fig. 3. (a) Ca XIX spectra syntesized from a flaring loop heated at the loop apex; the emission is integrated over the first 60 seconds from the beginning of the heating. (b) As in (a) but for heating at the base of the corona. (From Antonucci et al., 1987.) 

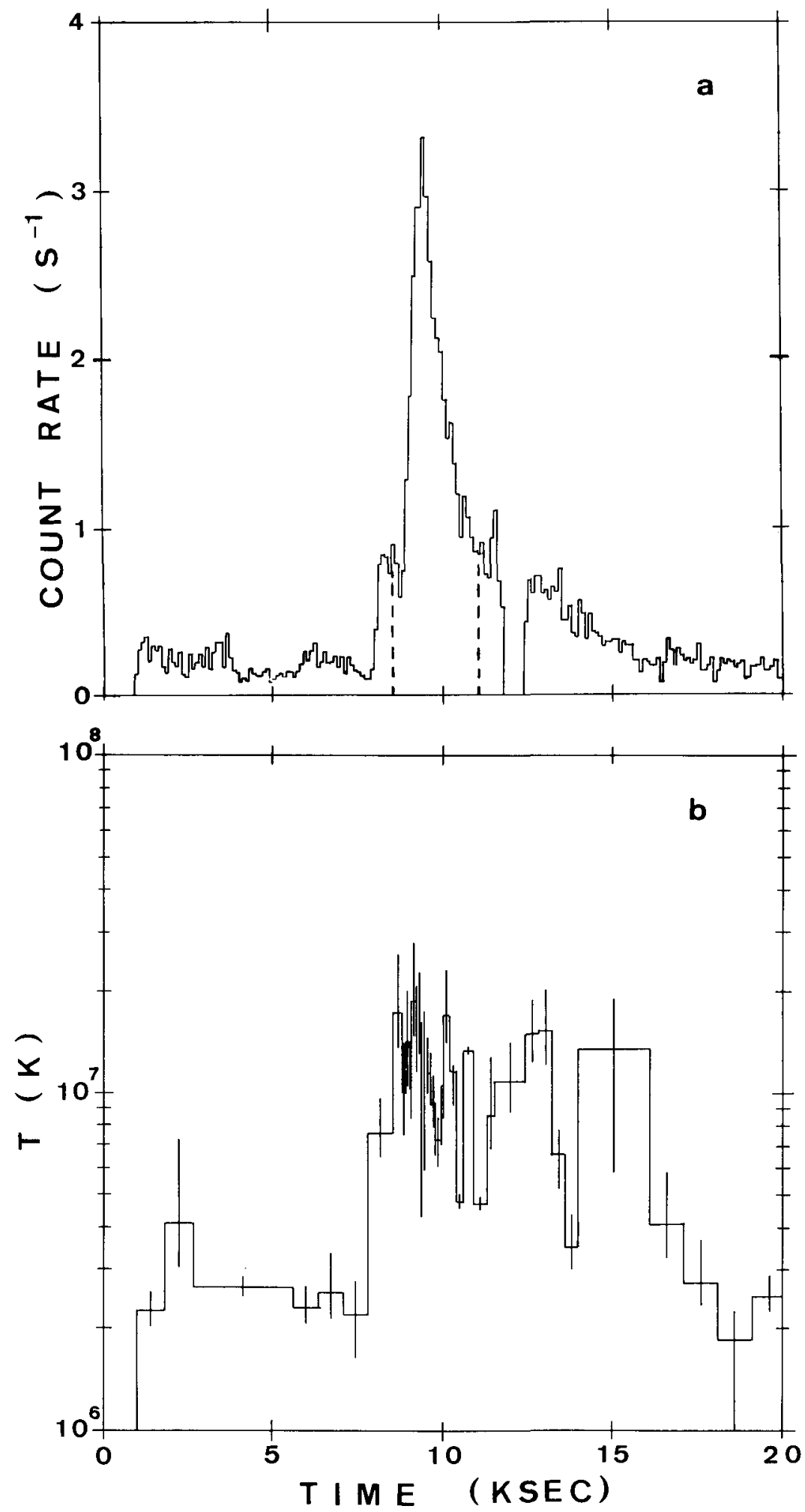

Fig. 4. (a) Observed light curve of the flare on Proxima Centauri detected in the IPC X-ray band with the Einstein satellite telescope (from Haisch et al. , 1983). The dashed vertical lines bound the time range covered by the hydrodynamic calculations. (b) Evolution of the single-component temperature, derived from the data. (From Reale et al., 1988.) 
Proportional Counter and Figure 4(b) reports the evolution of the plasma temperature derived from the data. The effective plasma temperature increases close to the flare maximum and then gradually decreases.

Observations of stellar flares lack all the information coming from spatial resolution; moreover, since the IPC is a wide band detector, the information from tracers of the heating, like the FexXV line at $1.85 \AA$, so useful for the solar simulations (Pallavicini et al., 1983, Peres et al., 1987), is missing. Part of our initial effort, therefore, has been to estimate from the observed features, and to extrapolate from the solar experience, a plausible set of pre-flare conditions, as well as the features of the heating. We have concentrated on local thermal heating, because the data of the Monitor Proportional Counter of the Einstein telescope do not show any clear evidence of a nonthermal hard $\mathrm{X}$-ray burst, a tracer of nonthermal beams of energetic electrons.

We have simulated the flare assuming three different values of loop semi-length $L$ : $10^{10} \mathrm{~cm}, 7 \times 10^{9} \mathrm{~cm}$ and $5 \times 10^{9} \mathrm{~cm}$. Figure 5 shows the light curve synthesized from the three simulations and evidences that the one with $L=7 \times 10^{9} \mathrm{~cm}$ provides the best

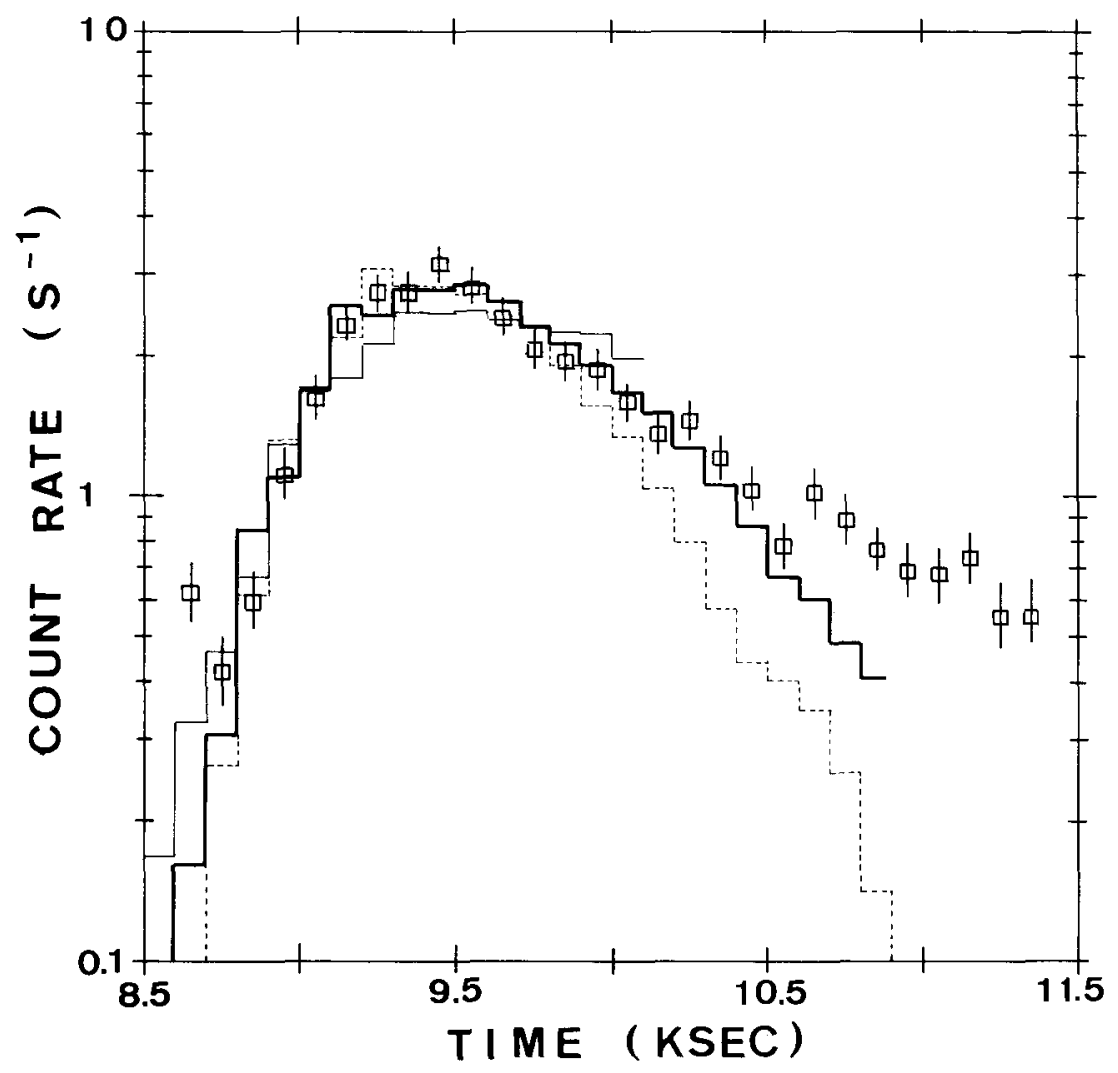

Fig. 5. Comparison between the Proxima Centauri flare light curve observed with the Einstein satellite telescope in the IPC X-ray band (squares) and the computed light curves for flare loops of semi-length $L=10^{10} \mathrm{~cm}$ (thin solid line), $L=7 \times 10^{9} \mathrm{~cm}$ (heavy), and $L=5 \times 10^{9} \mathrm{~cm}$ (dashed) and subjected to a heating flux pulse of $F=1.25 \times 10^{10} \mathrm{erg} \mathrm{cm}^{-2} \mathrm{~s}^{-1}$ lasting $700 \mathrm{~s}$. (From Reale et al., 1988.) 
fit to the observations. We have assumed that the impulsive heating is active at a constant intensity for the first $700 \mathrm{~s}$ of the flare and inactive afterwards. Under this assumption the decay phase allows the diagnostics of the loop length. The sensitivity of the results to the adopted value of $L$, evident in Figure 5, provides a means of establishing the accuracy of the value derived. The simulation with $L=10^{10} \mathrm{~cm}$ was interrupted at a time earlier than the other two because the computed decay time was evidently too long relative to the observed one. For further details see Reale et al. (1988).

Our approach shows that the model, used in conjunction with observations, can help to constrain physical quantities, like linear dimensions and coronal magnetic fields, not directly observable. For example, in the case of the Proxima Centauri flare, the hypothesis of confinement yields the constraint $B \geq 100 \mathrm{G}$.

\section{Conclusions}

The Palermo-Harvard model has proved to describe fairly well the gradual phase of solar flares by means of the detailed simulation of the light curves in the XRP lines (Pallavicini et al., 1983; Peres et al., 1987); it is also providing the fine diagnostics of the impulsive phase through the detailed simulation of high resolution spectra of the CaXIX (3.17 ̊) line (Antonucci et al., 1987).

Stellar studies are a relatively recent extension. The stellar observations cannot provide as stringent constraints as in the solar case; however, the extension is important because the range of physical parameters involved in the stellar flares is much wider than for solar flares alone. Stellar simulations allow physical insight and constraints on the dimensions and physical parameters not directly observable.

All these simulations show that the hydrodynamic and thermodynamic evolution of the confined plasma is a fundamental physical phenomenon of the gradual phase of compact loop flares. The fine tuning of the models to reproduce the observations typically provides the diagnostics of other physical aspects of the flare.

Many improvements have been proposed for these models. For instance they should use, in the transition region, the appropriate resolution of the numerical grid and, more importantly, a better treatment of the physics. In particular thermal conduction determines the steepness of the transition region and the structure of the whole atmosphere, thus imposing the requirements on the spacing of the numerical grid. However, the Spitzer (1962) formulation of heat flux, commonly used in these models, is not always appropriate because the mean free path of high speed electrons is often too long, with respect to the scale heights of temperature and density. A non-local treatment of thermal conduction would, instead, be more appropriate (Smith, 1986; Peres, Serio, and Rosner, 1987; Karpen and DeVore, 1987). In any case the computed evolution of the flare does not appear to be affected by the poor resolution (few tens of $\mathrm{km}$ ) in the transition region (e.g., Reale et al., 1988). An even more radical improvement would be to provide for a changing geometry, in order to model non-compact flares.

The interest in improvements shows the liveliness of this field. In fact hydrodynamical models of loop are now established theoretical and diagnostic tools in solar research and they promise to be useful also in stellar research. 


\section{Acknowledgements}

I thank S. Serio and G. S. Vaiana for stimulating discussions, F. Reale for helpful criticism, and the organizers of the meeting, B. Haisch and M. Rodonò, for their kind invitation. This work was supported by Ministero della Pubblica Istruzione and by Piano Spaziale Nazionale.

\section{References}

Acton, L. W., Culhane, J. L., Gabriel, A. H., and 21 co-authors: 1980, Solar Phys. 65, 53.

Antonucci, E., Gabriel, A. H., and 7 co-authors: 1982, Solar Phys. 78, 107.

Antonucci, E., Dodero, M. A., Peres, G., Serio, S., and Rosner, R.: 1987, Astrophys. J. $322,522$.

Giacconi, R. and 30 co-authors: 1979, Astrophys. J. 230, 540.

Haisch, B. M., Linsky, J. L., Bornmann, P. L., Stencel, R. E., Antiochos, S. K., Golub, L., and Vaiana, G. S.: 1983, Astrophys. J. 267, 280.

Karpen, J. T. and DeVore, C. R.: 1987, Astrophys. J. 320, 904.

Kopp, R. A., Fisher, G. H., MacNeice, P., McWhirter, R. W. P., and Peres, G.: 1986, in M. R. Kundu and Woodgate (eds.), Energetic Phenomena on the Sun, NASA Conference Publication 2439.

MacNeice, P., Pallavicini, R., Mason, H. E., Simnett, G. M., Antonnucci, E., Shine, R. A., Rust, D. M., Jordan, C., and Dennis, B. R.: 1985, Solar Phys. 99, 167.

Nagai, F. and Emslie, A. G.: 1984, Astrophys J. 279, 896.

Pallavicini, R., Serio, S., and Vaiana, G. S.: 1977, Astrophys. J. 216, 108.

Pallavicini, R. and Vaiana, G. S., Kahler, S. W., and Krieger, A. S.: 1975, Solar Phys. 45, 411.

Pallavicini, R., Peres, G., Serio, S., Vaiana, G. S., Acton, L., Leibacher, J., and Rosner, R.: 1983, Astrophys. J. 270, 270.

Peres, G., Rosner, R., Serio, S., and Vaiana, G. S.: 1982, Astrophys. J. 252, 791.

Peres, G., and Serio, S.: 1984, Mem. Soc. Astron. Ital. 55. 749.

Peres, G., Serio, S., and Rosner, R.: 1987, Nuovo Cimento B99, 29.

Peres, G., Reale, F., Serio, S., and Pallavicini, R.: 1987, Astrophys. J. 312, 895.

Reale, F., Peres, G., Serio, S., Rosner, R., and Schmitt, J. H. M. M.: 1988, Astrophys. J. $328,256$.

Rosner, R., Golub, L., and Vaiana, G. S.: 1985, Ann. Rev. Astron. Astrophys. 23, 413.

Smith, D. F.: 1986, Astrophys. J. 302, 836.

Spitzer, L.: 1962, The Physics of Fully Ionized Gases, Interscience, New York.

Vaiana, G. S. and Rosner, R.: 1978, Ann. Rev. Astron. Astrophys. 16, 393. 